\title{
Analyzing Spur-Distorted Impedance Spectra for the QCM
}

\author{
Sae Moon Yoon, ${ }^{1}$ Nam Joon Cho, ${ }^{2}$ and Kay Kanazawa ${ }^{2}$ \\ ${ }^{1}$ Electrical Engineering Department, Columbia University, 1300 S. W. Mudd, 500 West 120th Street, New York, NY 10027, USA \\ ${ }^{2}$ Department of Chemical Engineering, Stanford University, Stanford, CA 94305, USA
}

Correspondence should be addressed to Kay Kanazawa,procmon@msn.com

Received 22 December 2008; Revised 17 March 2009; Accepted 26 April 2009

Recommended by Michele Penza

The quartz crystal microbalance (QCM) is a sensitive device for determining the properties of materials loading it. One of the fundamental means of making these measurements is the so-called impedance (or admittance) spectra method. The resonant properties of the piezoelectric QCM sensor are measured over a spectral range in the neighborhood of a resonance, both with load and without load. The changes in the spectrum upon loading can be compared to models that describe the changes based on the mass density, the shear modulus, and the viscosity of the load. This comparison can be made with confidence so long as the spectrum corresponds to the model assumption of a single main resonance. Often, there exists a spurious resonance lying above the main resonance which is not included in the models. This can change the shape of the spectrum in ways not included in the model analysis. We describe a method we have used that separates the spur resonance from the main resonance, permitting the parameters of the main resonance to be isolated from that of the spur. These corrected parameters of the main resonance can then be used with confidence in model analyses.

Copyright (C) 2009 Sae Moon Yoon et al. This is an open access article distributed under the Creative Commons Attribution License, which permits unrestricted use, distribution, and reproduction in any medium, provided the original work is properly cited.

\section{Introduction}

1.1. Basic Description. The quartz crystal microbalance (QCM) has become an important tool for monitoring in many types of nanolayer assemblies of organic and biological films. A nice overview for its use in biomolecular interactions is given by Cooper and Singleton [1]. The QCM sensor is a resonant piezoelectric device and is described in a recent book [2]. It can resonate at its fundamental frequency and also at its odd multiple harmonics as discussed in a review by Buttry and Ward [3]. It consists of a disk with electrodes on opposing faces. When a load is placed upon the quartz crystal, changes in its resonant behavior permit an evaluation of certain properties of the load. A variety of electronic methods can be used to determine these changes, with some of them nicely given by Arnau [4]. This has made it very valuable not only to determine the amount of material in the load, but additional load properties such as stiffness and viscosity as well. Fortunately, the resonance behavior has been shown to be accurately represented by a simple equivalent circuit permitting the use of sophisticated electronic techniques to determine changes in the resonant properties. The full electrical equivalent for the QCM has been shown to be described by a so-called transmission line model [5] which can be simplified under conditions near a high $Q$ resonance to the simple equivalent circuit as discussed by Cernosek, Martin, Hillman, and Bandey [6]. The equivalent circuit is called the Butterworth Van Dyke (BVD) circuit $[7,8]$ and is shown in Figure 1.

The elements of the right hand branch contain the motional information. The changes due to loading can be directly incorporated as changes in these elements. The changes due to loading can be incorporated simply as changes in the values of the three elements in the right hand branch. An alternate method is to preserve the values of three elements, taking them to be the value corresponding to the unloaded crystal. The changes can be incorporated as small additional elements and this allows one to focus on the changes.

1.2. Three Key Methods of Study. The three most common methods for studying the resonant behavior of the QCM are (A) using the sensor as the active element in an electronic oscillator, (B) examining the transient decay of the current 




FIGURE 1: The Butterworth Van Dyke equivalent circuit.

after initial excitation of the sensor, and (C) recording the spectral behavior and its changes. The oscillator method is the most economical and is thus quite popular. From the measurement, it can determine the resonant frequency of the BVD circuit. More recently, it has become possible to also obtain the series resistance $R$. Generally, this is restricted to a single harmonic, although recently a dual harmonic oscillator has been described [9]. Two of the key properties of the resonance are its resonant frequency and the loss or energy dissipation. in the resonance. Using the oscillator approach, two of the properties of the circuit can be determined at a given harmonic, the resonant frequency $f_{N}$ and the resistance $R_{N}$.

The transient decay method [10] excites the QCM at its resonant frequency, then shorts the crystal and records the time behavior of the decaying current. This has been designed such that the decay currents for the various harmonics can be sequentially recorded. From the current decay for each harmonic, it is possible to determine the resonant frequency and the decay time. The decay time is related to the dissipation. Again, two of the important properties at each harmonic resonance are determined, in this case the resonant frequency $f_{N}$ and the dissipation $D_{N}$. These are discussed in more detail by Rodahl, et al. [11]. The ability to examine the behavior of multiple harmonics has been shown to be useful for interpreting the properties of the load. Although coming at an increased cost, it has shown itself to be extremely valuable in data analysis, particularly when studying viscoelastic loads.

Of primary interest in this paper is the study of the changes in the spectral behavior of the BVD circuit. This has come to be known as the "impedance" method and is used extensively. There have been a number of creative methods for determining the parameters of the load from spectral data, which demonstrates the versatility and flexibility of the impedance method. One, for example, is described by Berg et al. for the special case [12] in which an oscillating surface of quartz contacted an adjacent sphere. A somewhat different procedure was developed by Wang et al. [13] to use the admittance measurements to study gel swelling and deswelling. Another unusual application involves the use of impedance analysis for the measurement [14] of microliter volumes of liquid drops placed onto the crystals. The possibility of a scanning QCM was demonstrated by

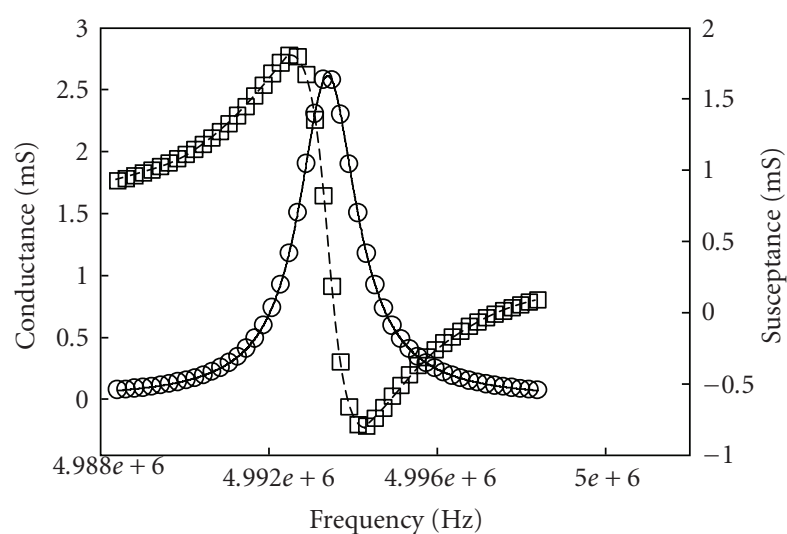

- Calculated conductance
$\bigcirc \quad$ Experimental conductance
--- Calculated susceptance
$\square \quad$ Experimental susceptance

FIGURE 2: The experimental points for a $5 \mathrm{MHz}$ fundamental crystal in water are illustrated by the circles (conductance) and squares (susceptance). The spectrum of the calculated 4 element BVD circuit is shown by the solid and dashed lines.

Oyama et al. [15], where scanning resolutions of 1 to $2 \mathrm{~mm}$ resolutions were found possible. There are many other nonstandard uses of QCM impedance analysis, which demonstrate that it is a very useful technique for many applications.

However, we focus here on the standard techniques and the possible degradations of the experimental data due to spurs. We consider the standard method to mean that the impedance being studied is that of a flat, AT-cut quartz disc with planar loads that can be modeled using a onedimensional analysis. Electrically, the resonant behavior can be summarized in terms of the admittance of the BVD circuit, which is the ratio of the current to applied voltage. This is the inverse of the impedance. A common method is to show the real part of the admittance ( the conductance $G$ ) or the imaginary part of the admittance (the susceptance $B$ ) as a function of the frequency. These are shown in Figure 2 with the units of $G$ and $B$ being the millisiemen (mS). The spectra for various harmonics can be recorded allowing for the study of multiple harmonics in this case as well. The ability to make a full determination of all three of the motional parameters in the BVD circuit drives much of the interest in this methodology.

The experimental data shows the behavior of a $5 \mathrm{MHz}$ crystal in deionized $18 \mathrm{M} \Omega \cdot \mathrm{cm}$ water. The calculated values were obtained by fitting the data to the BVD circuit. Although the fit is not perfect, it is very good and demonstrates the validity of the BVD representation. The resonant frequency is identified as the frequency of maximum conductance. The width of the peak is related to the loss, or dissipation of the resonance.

Another informative view of the admittance spectrum is a plot of the conductance along the abscissa and the susceptance along the ordinate, as shown in Figure 3. 


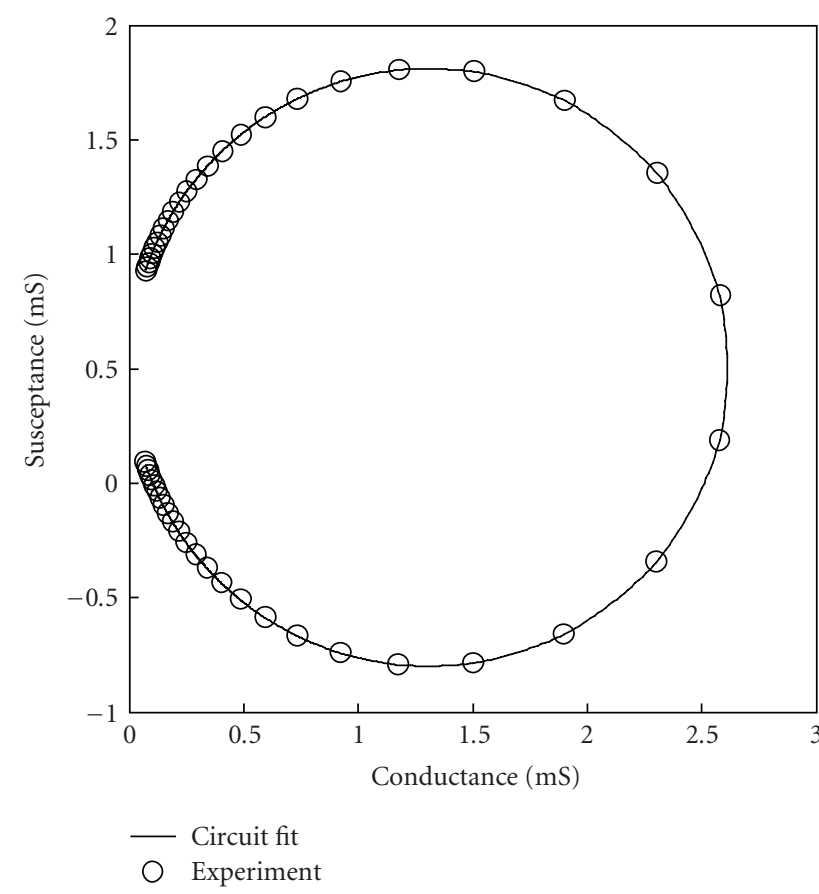

Figure 3: Admittance circle showing the circular form when the abscissa and ordinate scaling are identical.

If the same units are used on both axes, then it can be shown that the admittance plot is a circle. The diameter of the circle is the maximum conductance value. The circle center lies above the zero value of the ordinate and is due to the parallel capacitance $C_{P}$.

The agreement of the BVD description with experimental results shows that we can use these representations of the resonance to discuss the fitting procedure. There are sources that can distort these BVD diagrams and are discussed in the following section.

1.3. Deviations from the $B V D$. If a sufficiently large frequency span is taken for the spectrum, spurious resonances become visible. These are nicely described by Bottom in his classical text [15]. Taking the conductance data for several harmonics of the crystal in water, we observe, for example, as shown in Figure 4, the presence of an additional peak. The abscissa has been taken to be the index of the frequency plot. The frequency itself could not be used because it covers several harmonic ranges.

The additional resonance seen to the high frequency side of the main resonance is called a spurious resonance, or spur. The spur overlaps the main spectral peak giving rise to a distortion. We will show that the spur can be fully integrated into the fitting scheme to eliminate this source of distortion. The information in the susceptance data is better seen using the circular admittance plot.

The large admittance circles for the main resonances are visible. In addition, the smaller admittance circles for the spurs are also visible. The effects of the parallel capacitance,

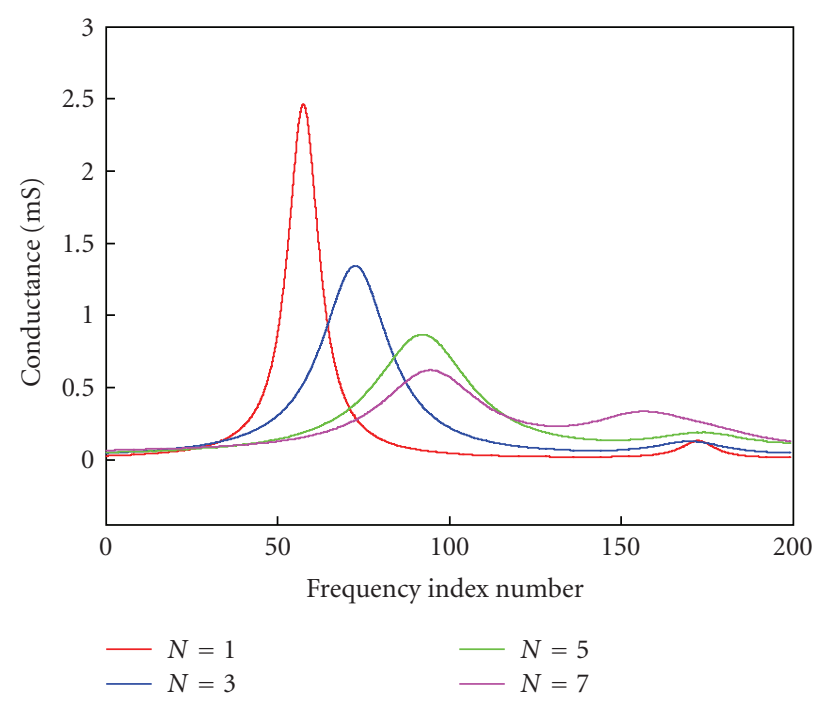

FIGURE 4: The conductance spectra are shown for several harmonics in the case of the quartz crystal having one face immersed in water.

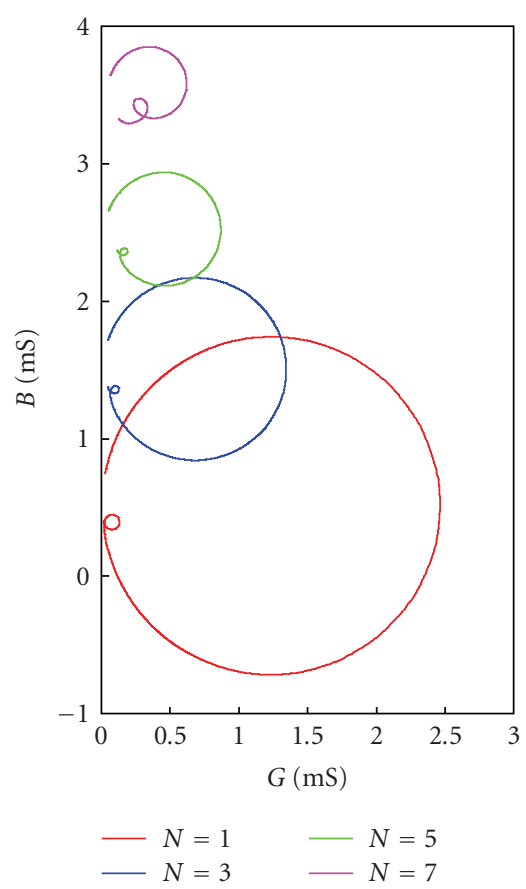

FIGURE 5: The circular admittance spectra over several harmonics.

$C_{P}$, are seen as the offset of the circles' centers along the ordinate. This vertical offset increases for increasing harmonic resulting from the increasing capacitive susceptance with increasing frequency. This capacitance reflects the dielectric capacitance of the quartz disk as well as the capacitance of wires and cables used to connect the crystal. The constancy of this capacitance is clearly seen if we subtract a susceptance value corresponding to $16 \mathrm{pf}$ from each admittance circle. In that case, we then observe Figure 6.

In addition to the capacitance effect of $C_{P}$, we can also note that the admittance circles are not tangential to the 


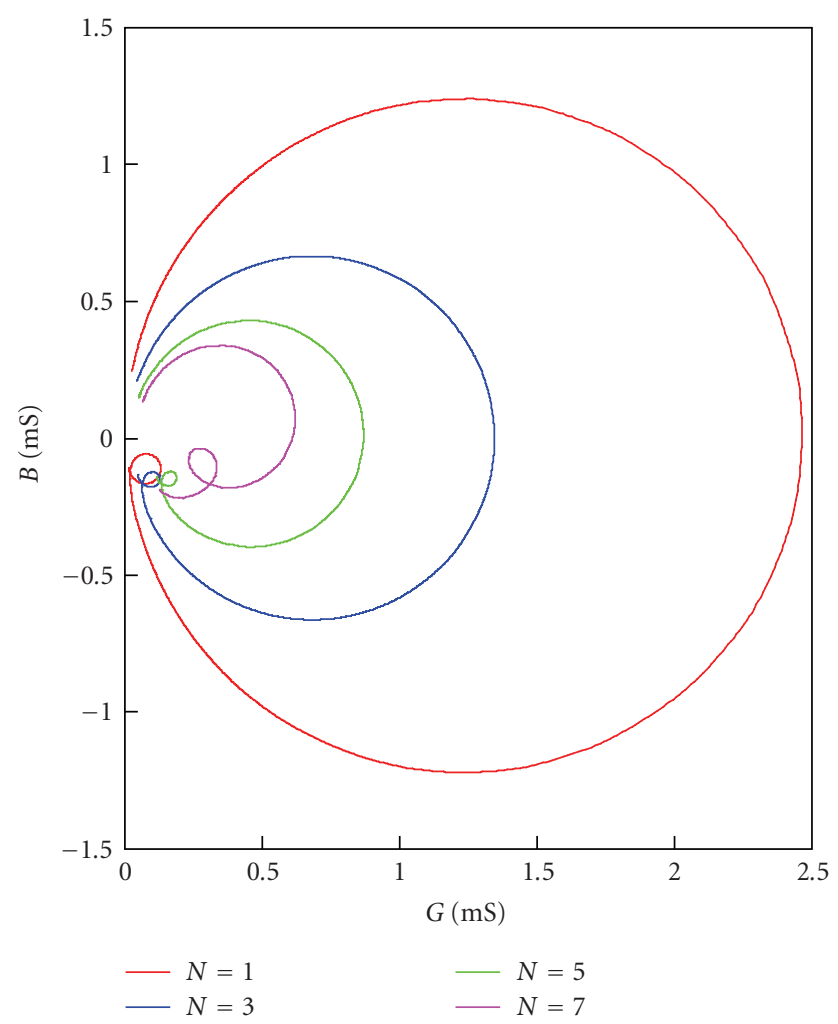

FIgURE 6: The admittance circles corrected for a constant $16 \mathrm{pf}$ capacitance.

ordinate as would be expected from the simple BVD circuit. There is a horizontal offset that increases with increasing harmonic. This is not a large effect, but its effect can be described by the presence of a frequency dependent conductance in parallel with the BVD circuit. See, for example, the recognition and description of such a parallel path by Lucklum et al. [16].

These deviations are easily summarized in the circular admittance plots. The capacitance $C_{P}$ gives rise to a vertical shift in the circle. The spurs are evident from the extra circle on the high frequency end, and a parallel conductive loading appears as a shift of the circle to the right.

\section{Experimental Details}

To obtain the experimental data used for fitting, we used quartz crystals obtained from Maxtek, Inc. The crystals had a diameter of 1 inch and were plano-plano $5 \mathrm{MHz}$ AT cut crystals having asymmetrical electrodes. The thicknesses are in the range of $331 \mu \mathrm{m}$. The smaller electrode defined the common areal region and had an areal value of $3.419 \times$ $10^{-5} \mathrm{~m}^{-2}$. The crystals were placed in the standard polyvinyl chloride holder was provided by Maxtek. The glass cell provided by Maxtek was used. The glass cell and the circular crystal holder are shown in Figures 7(a) and 7(b).

The PVC crystal holder shown in Figure 7(b) contained the crystal and was connected to the connector shown on the glass portion of the cell. O-rings sealed the crystal on both

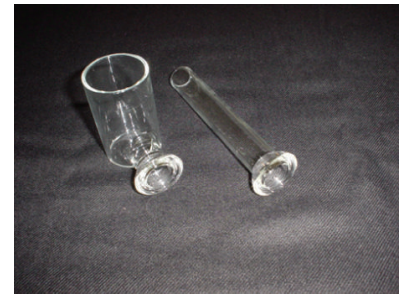

(a)

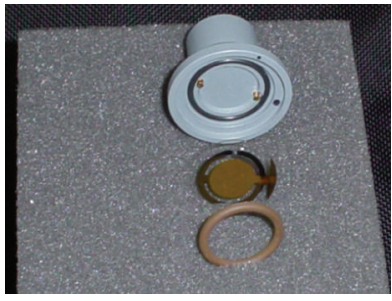

(b)
Figure 7: (a) Two types of glass cells are shown. The one on the left was used in this study. The PVC crystal holder is shown with the black O-ring. (b) The crystal is shown In front of the holder along with the sealing O-ring.

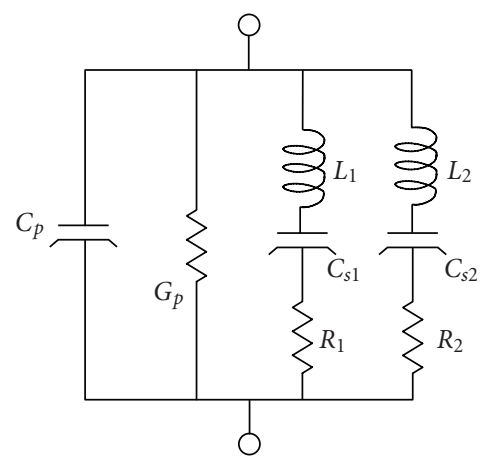

FIGURE 8: The equivalent circuit used at each harmonic.

sides. The holder was held to the cell by a spring clip. The circular crystal holder terminated in an SMB connector. To connect this unit to the Agilent 4291-A Impedance probe, it was necessary to use an approximate 1 foot length of SMB cable, and then an SMB to BNC adapter. The instrument used for capturing the spectra was the Agilent 4294 Precision Impedance Analyzer.

The crystal was cleaned by immersion in an ethanol solution and drying with a gentle stream of nitrogen. It was then placed in an oxygen plasma unit. Deionized water of $18 \mathrm{M} \Omega \cdot \mathrm{cm}$ purity was used in the glass cell.

\section{Fitting Procedure}

The experimental data was treated in a fitting procedure designed to extract the values of the resonance properties. In the ideal case, the circuit shown in Figure 1 is appropriate. In such a case, the spectrum is described by a Lorentzian shape and a fit to this shape would be appropriate. For the high quality (low dissipation) circuits occurring with the QCM, the Lorentzian spectra exhibit a symmetry about the resonant frequency. As we have seen, spurs can distort the symmetry of the resonance. In addition we have also seen that the parallel capacitance $C_{P}$ can displace the Lorentzian circles vertically and shunt conductances can displace them horizontally.

To incorporate the various aspects of the equivalent circuit, we use the circuit shown in Figure 8. 
$C_{P}$ is the parallel capacitance, including all of the connecting adapters and cables. $G_{P}$ represents the effects due to a conductive shift of the circle to the right. The branch with the subscript 1 represents the motional aspects of the main resonance of the quartz crystal, while that with the subscript 2 represents the resonant spur. We can write the admittance in terms of the individual circuit elements shown above as

$$
\begin{aligned}
Y= & G+j B \\
= & G_{P}+j \omega C_{P}+\frac{1}{j \omega L_{1}+\left(1 / j \omega C_{S 1}\right)+R_{1}} \\
& +\frac{1}{j \omega L_{2}+\left(1 / j \omega C_{S 2}\right)+R_{2}} .
\end{aligned}
$$

However, we have used an alternate expression for this same admittance in terms of parameters more convenient for the fitting procedure, and is shown below:

$$
\begin{aligned}
Y= & G_{P}+j \omega C_{P}+\frac{G 1_{\max }}{1+j(1 / D 1)\left(\left(f / f_{10}\right)-\left(f_{10} / f\right)\right)} \\
& +\frac{G 2_{\max }}{1+j(1 / D 2)\left(\left(f / f_{20}\right)-\left(f_{20} / f\right)\right)} .
\end{aligned}
$$

The values used instead of $L, C_{S}$, and $R$ are $G_{\max }, D$, and $f_{0}$. $G 1_{\max }$ is the maximum conductance of the main resonance, $D 1$ is the dissipation of the main resonance peak and $f_{10}$ is the resonant frequency of the main resonance. Dissipation is simply the inverse of the more familiar quality factor or $Q$ of the circuit. It is treated in more detail by Rodahl et al. [10]. Relations connecting the parameters are as follows:

$$
\begin{aligned}
& G 1=\frac{1}{R 1}, \\
& f_{10}=\frac{1}{2 \pi \sqrt{(L 1)\left(C_{S 1}\right)}}, \\
& D 1=\frac{R 1}{2 \pi f_{10} L 1} .
\end{aligned}
$$

These connect the parameters describing the main resonance branch. There are a set of equivalent connecting equations for the spurious resonance branch. In order to fit the experimental spectra to (2), it is necessary to provide some "guess" values for these parameters. The experimental spectra are a record of the conductance and the susceptance over a range of frequencies. It is possible to obtain spectra with the number of frequencies in the range of 50,100, 200, or 400 or more. We typically used the spectra consisting of 400 frequency values. We generated a subroutine with which we obtained starting guess values from the experimental spectra. The guess value of $G_{\max }$ is obtained by recording the maximum value of $G$ over a resonance. The frequency at which this occurs is taken as the guess value for the resonant frequency $f_{0}$. To obtain a guess value for the dissipation is slightly more complex. We find the maximum value of the susceptance in the resonance region and take its frequency $f_{\mathrm{Bmax}}$. We calculate the frequency difference

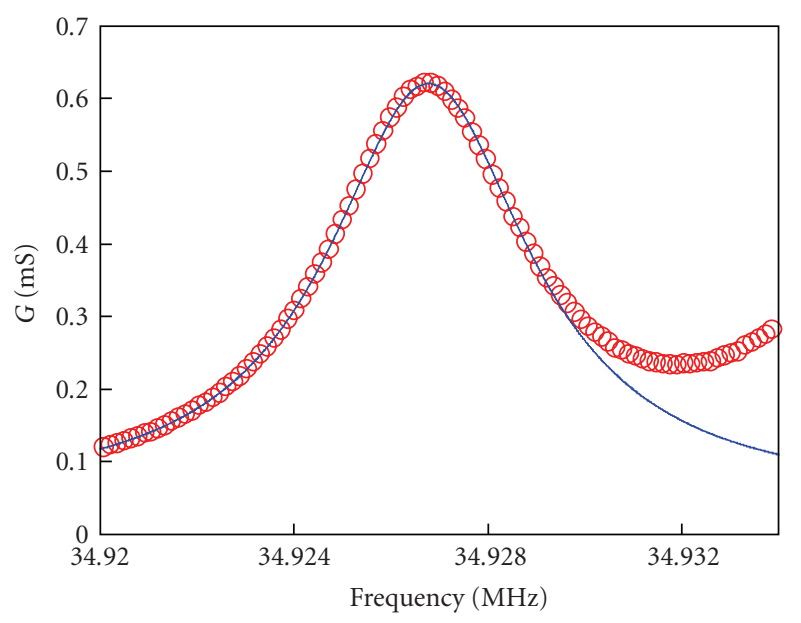

Experimental data

— Main resonance only

FIGURE 9: The calculated fit using only the main resonance (blue) with the experimental data shown in red circles.

$\Delta f=\left(f_{0}-f_{\mathrm{Bmax}}\right)$. Twice this value is taken as the full width at half maximum for the resonance. The guess value for the dissipation $D$ is then taken to be $(2 \Delta f / f 0)$. To fit a single main resonance (without spur), 5 guess values are required; $C_{P}, G_{P}, G 1_{\max }, f_{10}$, and $D 1$. For fitting to a main resonance with a spur, three additional guess values are required: $G 2_{\max }, f_{20}$, and $D 2$.

While there are many other possible ways to do the fitting,we chose to use the method called "genfit" in the Mathcad program. It is described in their literature as a leastsquares nonlinear regression. For those more familiar with these methods, they also describe it as using a LevenbergMarquardt approach to the minimization.

In beginning this experiment, we were not certain that the spur resonance could be included in the fit to a resonance. It was possible, for example, that the threeelement equivalent circuit for the spur was too simplistic, or it was possible that the fitting procedure would not permit the inclusion of the large number of variables. We examined this possibility by an initial demonstration. For this demonstration, we have taken one case of the data for the seventh harmonic and compare the results of the fitting with and without spur inclusion.

In Figure 9, the main resonance of the conductance at the seventh harmonic is shown. The fits are made to the conductance data. When the spur effect is neglected, a careful look at the spectrum indicates that the experimental curve does not have the symmetrical shape expected for a single resonance. Even a possible shift in the position of the peak is suggested. We believe this to be the result of the overlap of the spurious resonance with the main resonance. We include the first spur of the seventh harmonic and obtain the following results.

Again, the fits were made using only the conductance data with the guess values being derived from the conductance spectrum as described earlier. The fit to the susceptance 


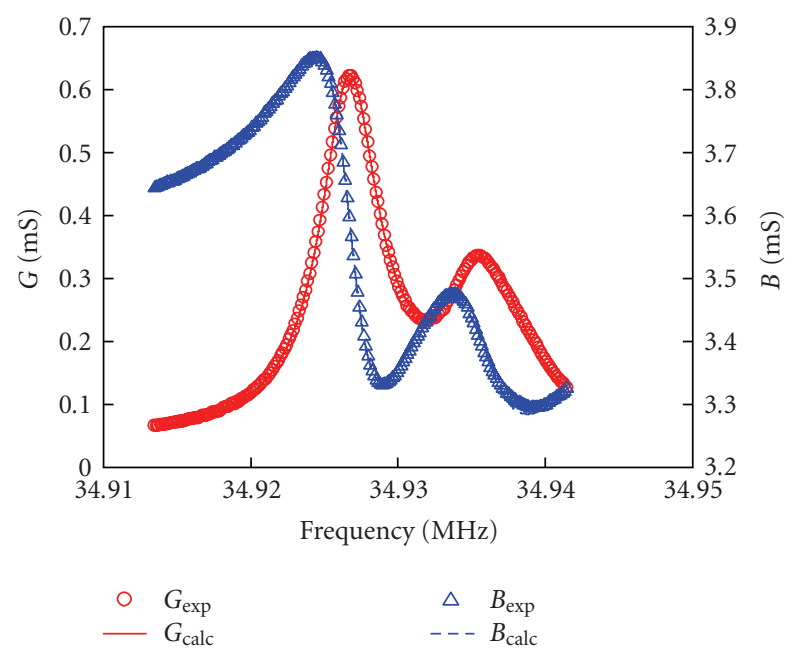

FIgURE 10: The seventh harmonic data are shown including the first spur. The experimental $G$ and $B$ values are shown by the red circles and the blue triangles, respectively. The calculated fits using the equivalent circuit of Figure 8 are shown by the solid red line and dashed blue line.

TABLE 1: Values of circuit parameters without correcting for spur (black) and with correcting for the spur (red).

\begin{tabular}{cccc}
\hline$N$ & $\begin{array}{c}\text { Fmain } \\
\text { MHz }\end{array}$ & $\begin{array}{c}\text { Gmain } \\
\mathrm{mS}\end{array}$ & $\begin{array}{c}\text { Dmain } \\
10^{-6}\end{array}$ \\
\hline 1 & 4.993348 & 2.45958 & 353.316 \\
& 4.993348 & 2.46127 & 352.953 \\
3 & 14.970878 & 1.33199 & 188.701 \\
& 14.970874 & 1.33314 & 187.991 \\
5 & 24.949092 & 0.844077 & 155.258 \\
& 24.949080 & 0.839339 & 153.141 \\
7 & 34.936778 & 0.573590 & 145.474 \\
& 34.926700 & 0.549358 & 136.959 \\
\hline
\end{tabular}

data is also extremely good. The quality of the fits provided confidence in the fitting procedure. By including the effects of the spur resonance, we feel that we can be more assured of the accuracy of the elements representing the main resonance. The accuracy of the representation for the main resonance is important since the changes due to the load are calculated from the one-dimensional electromechanical model which represents the single main resonance.

\section{Results of the Fitting}

This same procedure was used to fit the 1 st, $3 \mathrm{rd}$, and 5 th harmonics as well. To show the changes incurred by including the spur, we have listed in Table 1 the values of the characteristics of the main resonance determined without the spur correction (black) and with the spur correction (red).

The differences do not appear to be large. However, we must remember that the changes induced by the additional
TABLE 2: Values of the BVD circuit at the various harmonics are given.

\begin{tabular}{cccc}
\hline$N$ & $R$ & $L$ & $C_{S}$ \\
& $\Omega$ & $H$ & $f F$ \\
\hline 1 & 406.57 & 0.03668 & 27.70 \\
& 406.29 & 0.03369 & 27.69 \\
3 & 750.76 & 0.04230 & 2.672 \\
& 750.11 & 0.04242 & 2.664 \\
5 & 1184.7 & 0.04867 & 0.8359 \\
& 1191.4 & 0.04963 & 0.8200 \\
7 & 1743.4 & 0.05461 & 0.3802 \\
& 1820.3 & 0.06056 & 0.3429 \\
\hline
\end{tabular}

TABLE 3: Values calculated from the one-dimensional theory.

\begin{tabular}{lccc}
\hline$N$ & Res. Freq. & Induct. & $C_{S}$ \\
& $\mathrm{~Hz}$ & $H$ & $f F$ \\
\hline 1 & 4999210 & 0.03886 & 26.0833 \\
3 & 15040717 & 0.03897 & 2.8736 \\
5 & 25073036 & 0.03897 & 1.0338 \\
7 & 35106021 & 0.03898 & 0.52733 \\
9 & 45137744 & 0.03898 & 0.31898 \\
11 & 55169313 & 0.03898 & 0.21352 \\
13 & 65200803 & 0.03898 & 0.11259 \\
\hline
\end{tabular}

TABle 4: Values of $C_{p}$ and $G_{p}$ fitted to the data for the harmonics.

\begin{tabular}{lccc}
\hline$N$ & $C_{p}(\mathrm{pf})$ & $G_{p}(\mu \mathrm{S})$ & $R p(K \Omega)$ \\
\hline 1 & 16.134 & 4.0271 & 248.32 \\
3 & 15.884 & 10.784 & 92.73 \\
5 & 15.909 & 22.985 & 43.507 \\
7 & 16.006 & 45.050 & 22.198 \\
\hline
\end{tabular}

mass of the film and liquid are very small. So it is necessary to obtain a relatively high accuracy for these values. Using (3) it is possible to obtain direct relations between the values of $f_{0}, G_{\max }$, and $D$ with $L, C_{S}$, and $R$. These results are shown in Table 2 in the more familiar values of the BVD circuit elements.

An unexpected feature of these results is that the inductance appears to change with the harmonic. While not a major point of study here, we include the values calculated from the one-dimensional theory for comparison. The inductance was found to be almost constant, independent of harmonic. The calculated values are shown in Table 3.

We extended the values to the 13th harmonic to ensure the constancy of the inductance with harmonic number. The calculated changes are very small, which made the results of the measurements somewhat surprising. This is perhaps worthy of further study, but was not pursued here.

While we have not dwelled on the corrections for the parallel capacitance and the parallel conductance, these were also used in the fitting algorithm. For interest, these values are shown in Table 4. 


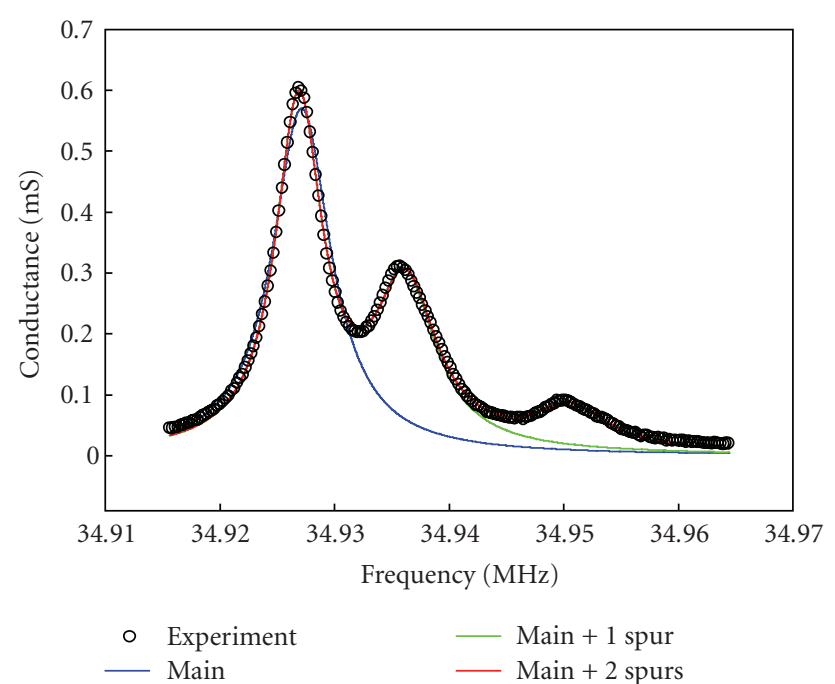

FIGURE 11: Fitting the conductance of the 7th harmonic containing two spurs.

As can be seen, the values for $C_{P}$ are almost constant indicating again the invariance of the dielectric parameters with frequency. The conductance values are included only to indicate that the horizontal shift in the admittance circle can be accounted for. Because resistance is a more familiar variable than conductance, we have converted the conductance to resistance, shown in the last column. Except to remark on the monotonic behavior, we make no further comment on this variable.

The successes obtained in fitting the main resonance, spurs and parallel admittances were very satisfying. In the case of the 7th harmonic, a second spur was seen above the first spur. We extended the treatment discussed above to have an additional three elements branch to describe a second spur and attempted a fit to see whether the fitting routine was robust enough to include additional variables. The results are shown in Figure 11.

The agreements fitting to only the main resonance (blue), to the main and first spur (green) and to the main and both spurs (red) are very satisfying.

\section{Conclusion}

One major method for determining the physical parameters of loads placed on the quartz crystal microbalance is the admittance (or impedance) method. The recorded values caused by changes in the electrical measurements of the admittance spectrum are fit to a model. A major perturbation that can distort the measured electrical spectrum is the presence of spurious resonances. In addition, perturbations arising from the connection of the crystal transducer to the measurement apparatus can also distort the spectrum. We have shown a method by which these three effects can be quantitatively accounted for, increasing the confidence in the accuracy of the fit.

\section{Acknowledgments}

The authors wish to thank Stanford University's Center for Polymer Inerfaces and Macromolecular Assemblies (CPIMA) for use of its facility. This work was completed through a CPIMA SURE program award to SMY.

\section{References}

[1] M. A. Cooper and V. T. Singleton, "A survey of the 2001 to 2005 quartz crystal microbalance biosensor literature: applications of acoustic physics to the analysis of biomolecular interactions," Journal of Molecular Recognition, vol. 20, no. 3, pp. 154-184, 2007.

[2] A. A. Vives, Piezoelectric Transducers and Applications, vol. 26, Springer, Berlin, Germany, 2008, 125 illustration.

[3] D. A. Buttry and M. D. Ward, "Measurement of interfacial processes at electrode surfaces with the electrochemical quartz crystal microbalance," Chemical Reviews, vol. 92, no. 6, pp. 1355-1379, 1992.

[4] A. Arnau, "A review of interface electronic systems for AT-cut quartz crystal microbalance applications in liquids," Sensors, vol. 8, no. 1, pp. 370-411, 2008.

[5] E. Benes, "Improved quartz crystal microbalance technique," Journal of Applied Physics, vol. 56, no. 3, pp. 608-626, 1984.

[6] R. W. Cernosek, S. J. Martin, A. R. Hilhnan, and H. L. Bandey, "Comparison of lumped-element and transmissionline models for thickness-shear-mode quartz resonator sensors," IEEE Transactions on Ultrasonics, Ferroelectrics, and Frequency Control, vol. 45, no. 5, pp. 1399-1407, 1998.

[7] S. J. Martin, V. E. Granstaff, and G. C. Frye, "Characterization of a quartz crystal microbalance with simultaneous mass and liquid loading," Analytical Chemistry, vol. 63, no. 20, pp. 22722281, 1991.

[8] H. Muramatsu, E. Tamiya, and I. Karube, "Computation of equivalent circuit parameters of quartz crystals in contact with liquids and study of liquid properties," Analytical Chemistry, vol. 60, no. 19, pp. 2142-2146, 1988.

[9] M. Ferrari, V. Ferrari, and K. Kanazawa, "Dual-harmonic oscillator for quartz crystal resonator sensors," Sensors and Actuators A, vol. 145-146, no. 1-2, pp. 131-138, 2008.

[10] M. Rodahl, F. Höök, A. Krozer, P. Brzezinski, and B. Kasemo, "Quartz crystal microbalance setup for frequency and Qfactor measurements in gaseous and liquid environments," Review of Scientific Instruments, vol. 66, no. 7, pp. 3924-3930, 1995.

[11] M. Rodahl, F. Höök, and B. Kasemo, "QCM operation in liquids: an explanation of measured variations in frequency and Q factor with liquid conductivity," Analytical Chemistry, vol. 68, no. 13, pp. 2219-2227, 1996.

[12] S. Berg, D. Johannsmann, and M. Ruths, "Frequency response of quartz crystal shear-resonator during an adhesive, elastic contact in a surface forces apparatus," Journal of Applied Physics, vol. 92, no. 11, pp. 6905-6910, 2002.

[13] Z. Wang, D. Kuckling, and D. Johannsmann, "Temperatureinduced swelling and de-swelling of thin poly $(\mathrm{N}$ isopropylacrylamide) gels in water: combined acoustic and optical measurements," Soft Materials, vol. 1, pp. 353-374, 2003.

[14] A. Saluja and D. S. Kalonia, "Measurement of fluid viscosity at microliter volues using quartz impedance analysis," AAPS PharmSciTech, vol. 5, pp. 1-14, 2004. 
[15] N. Oyama, T. Tatsuma, S. Yamaguchi, and M. Tsukahara, "Scanning electrode quartz crystal analysis," Analytical Chemistry, vol. 69, no. 6, pp. 1023-1029, 1997.

[16] R. Lucklum, G. Behling, R. W. Cernosek, and S. J. Martin, "Determination of complex shear modulus with thickness shear mode resonators," Journal of Physics D, vol. 30, no. 3, pp. 346-356, 1997. 

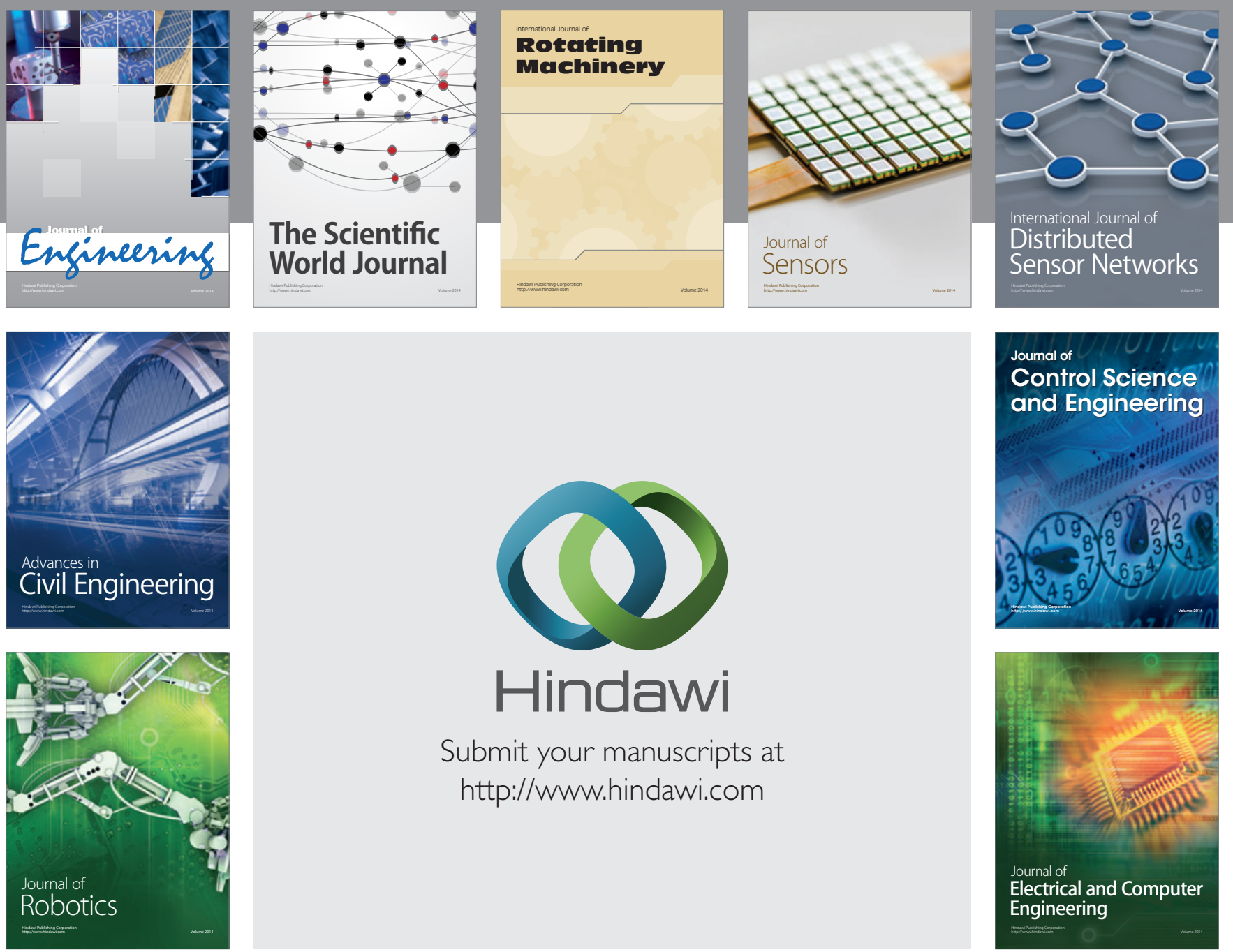

Submit your manuscripts at

http://www.hindawi.com
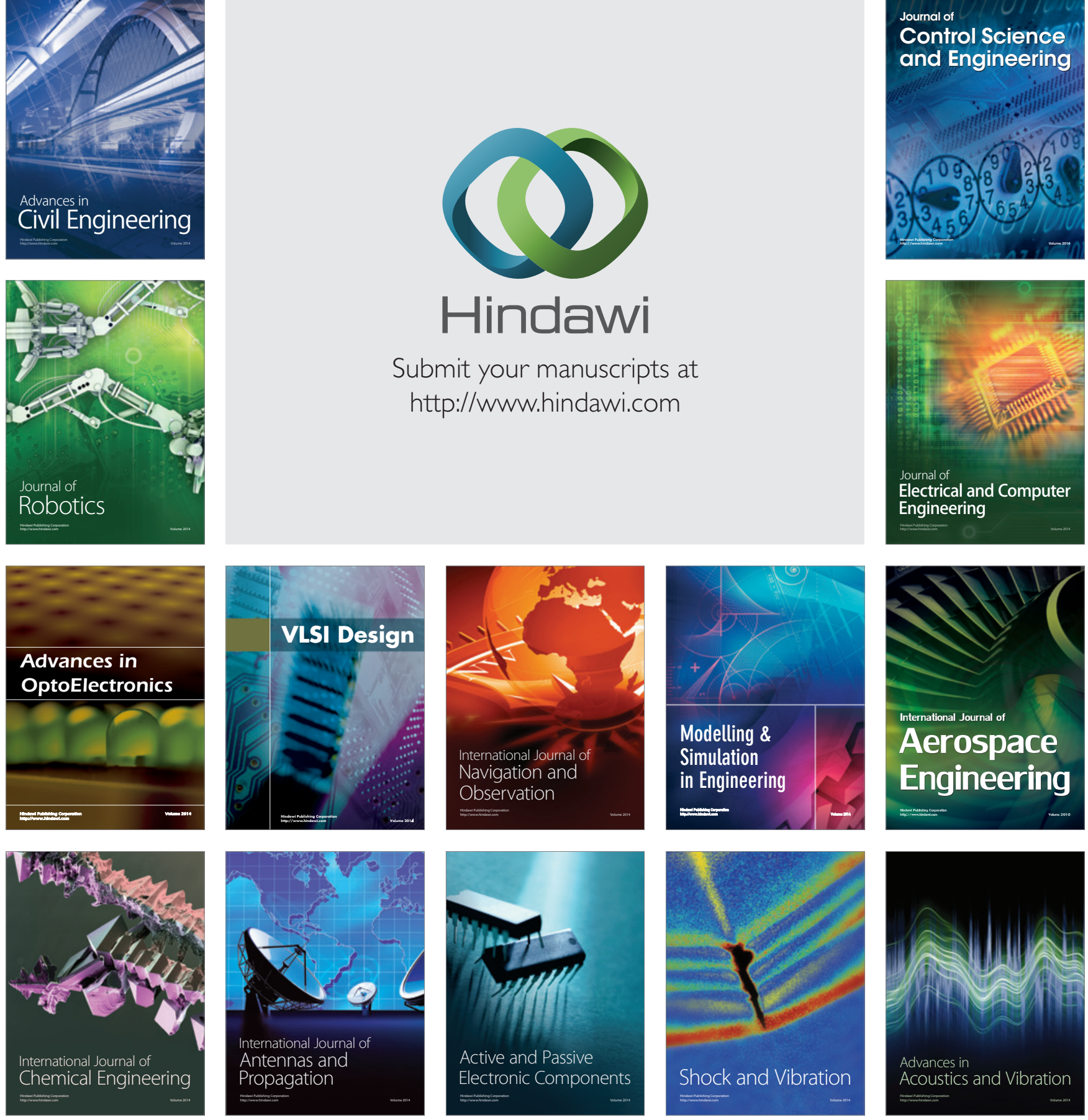